\title{
Desempenho regional do IDH e do PIB per capita dos municípios de Mato Grosso do Sul, Brasil, entre 2000 e 2010
}

\author{
Performance of HDI and per capita GDP regional of municipalities of Mato Grosso \\ do Sul, Brazil, between 2000 and 2010
}

\section{Performance de HDI régional et PIB par habitant des municipalités du Mato Grosso do Sul, Brazil, entre 2000 à 2010} \author{
Brazil, entre 2000 a 2010
Michel Constantino*
(michel@ucdb.br)
Alexander Bruno Pegorare*
(alexandre.pegorare@ibge.gov.br)
Reginaldo Brito da Costa*
(reg.brito.costa@gmail.com)

Desempeño del IDH y PIB per capita regional de municipios de of Mato Grosso do Sul,

Recebido em 10/10/2015; revisado e aprovado em 23/10/2015; aceito em 30/11/2015

DOI: http:/ /dx.doi.org/10.20435/1984042X2016207

\begin{abstract}
Resumo: Este artigo objetivou analisar a expansão e convergência do Índice de Desenvolvimento Humano (IDH) e do Produto Interno Bruto (PIB) per capita nos municípios de Mato Grosso do Sul no período do ano de 2000 a 2010. Os resultados mostraram que houve convergência e avanços socioeconômicos e que os mapas temáticos são importantes fontes de análise para políticas públicas locais e regionais.

Palavras-chave: Î́ndice de Desenvolvimento Humano; PIB per capita; Mato Grosso do Sul.

Abstract: This paper aimed to analyze the expansion and convergence of the Human Development Index and Gross Domestic Product per capita in the municipalities of Mato Grosso do Sul in the year 2000 to 2010. The results showed that there was convergence and socio-economic progress, and that thematic maps are important analytical sources for local and regional public policies.

Key words: Human development Index; GDP per capita; Mato Grosso do Sul.

Résumé: Cet article vise à analyser l'expansion et de la convergence de Indice de développement humain et le produit intérieur brut par habitant dans les municipalités de Mato Grosso do Sul, dans l'année 2000 à 2010. Les résultats ont montré qu'il y avait convergence et le progrès socio-économique, et que cartes thématiques sont des sources importantes d'analyse des politiques publiques locales et régionales.

Mots-clés: Indice de Développement Humain; PIB par habitant; Mato Grosso do Sul.

Resumen: Este trabajo tuvo como objetivo analizar la expansión y la convergencia del Índice de Desarrollo Humano y el Producto Interno Bruto per cápita en los municipios de Mato Grosso do Sul, en el año 2000 a 2010. Los resultados mostraron que no hubo convergencia y el progreso socioeconómico y mapas temáticos son fuentes importantes para el análisis de las políticas públicas local y regional.

Palabras clave: Índice de Desarrollo Humano; PIB per cápita; Mato Grosso do Sul.
\end{abstract}

\section{INTRODUÇÃO}

Segundo o Fundo Monetário Internacional (FMI, s.d.), no ano de 2010 o Brasil ficou em 6o lugar no ranking dos países com participação no Produto Interno Bruto (PIB), que é o valor final total de bens e serviços produzidos por uma nação em um dado ano; porém, quando comparado ao PIB per capita (que é o PIB dividido pela população desse mesmo ano), sua posição caiu para o $61^{\circ}$ lugar, traduzindo assim uma grande desigualdade social no país. Apesar disso, o PIB per capita do Brasil vem crescendo, entre os anos de 2000 a 2010 o PIB real teve um avanço de quase $102 \%$, influenciado principalmente pelo controle inflacionário e o aumento das exportações no período (IBGE, s.d.).

No Relatório de Desenvolvimento Humano 2011, lançado pela Organização das Nações Unidas (UNDP, s.d.), o Brasil em 2010 se encontrava na $85^{\mathrm{a}}$ posição no ranking global de IDH $(0,739)$, enquanto que no ano 2000, o país estava em $73^{\circ}$ lugar $(0,757)$. Ou seja, mesmo com a melhora de PIB per capita no período, houve uma queda no IDH de

* Universidade Católica Dom Bosco (UCDB), Campo Grande, Mato Grosso do Sul, Brasil. 
$4,25 \%$, queda que poderia ter sido mais grave caso não tivesse havido o aumento da renda por pessoa nesse período.

Nesse sentido, esses indicadores, quando divulgados e expressos apenas numericamente, dificultam uma visualização mais ampla dos problemas sociais e econômicos, impedindo, muitas vezes, uma análise para aplicação de soluções e políticas públicas locais e regionais.

A metodologia de representação por mapas temáticos é importante para verificar extensões territoriais com suas singularidades regionais. Em conjunto com os mapas temáticos, os testes de convergência são utilizados para apontar se os indicadores socioeconômicos de locais menos desenvolvidos estão evoluindo ao longo do tempo para os mais desenvolvidos.

O objetivo deste estudo foi representar e interpretar o impacto do IDH e do PIB per capita dos municípios de Mato Grosso do Sul (MS), tendo como base o período de 2000 a 2010. Pretendeu-se ampliar os conhecimentos sobre como a análise territorial dos dados socioeconômicos podem contribuir para uma explicação mais aprofundada dos aspectos relacionados à qualidade de vida da população, desenvolvimento econômico, e municiar informações para tomadores de decisões políticas.

O presente artigo, portanto, se compõe de quatro seções, sendo a primeira contemplada por esta introdução. A seção 2 com os aspectos metodológicos. Na seção 3, apresenta-se uma breve discussão de como o IDH e o PIB per capita repercutiram no Brasil, no estado de Mato Grosso do Sul e em seus municípios no período de 2000 a 2010, bem como as principais abordagens recentes da literatura sobre como foi estudado o tema. Por fim, a seção 4 destina-se a apresentar as conclusões do estudo.

\section{METODOLOGIA}

Foram utilizados os dados disponibilizados pelo Programa das Nações Unidas para o Desenvolvimento - Brasil e Internacional (PNUD, s.d.; UNDP, s.d.) e os dados oficiais do PIB municipal (Contas Nacionais), população municipal (Censo), da Pesquisa Agrícola Municipal (PAM) e da Pesquisa
Pecuária Municipal (PPM) do ano de 2000 e 2010, através do sistema de dados agregados do Sidra-IBGE (IBGE, s.d.).

Utilizou-se, para a estatística descritiva e para a elaboração dos mapas temáticos, o Programa computacional IpeaGeo 2.1.

Quando se comparam os PIBs em séries históricas, deve ser observado se o PIB divulgado é o PIB real (corrigido pela depreciação do poder de compra), ou se é o praticado a preços correntes (nominal). Assim, para este último deve-se utilizar o deflator do PIB para que seja possível sua comparação entre os anos. No cálculo do PIB per capita, como o IBGE disponibiliza somente o PIB a preços correntes, o PIB do ano de 2000 foi corrigido aos valores do ano de 2010 através do Índice de Preços ao Consumidor Amplo (IPCA), que o adequou à inflação oficial no período. Assim, nesse período, segundo o IBGE (s.d.), a inflação de janeiro de 2000 a dezembro de 2010 foi de 101,18\%.

Para calcular o PIB per capita municipal, foi utilizada seguinte fórmula:

$$
P I B C M=\frac{P I B R M}{P M}
$$

onde: $P I B C M=$ produto interno bruto per capita municipal (R\$);

PIBRM = produto interno bruto real municipal (R\$);

$P M=$ população residente municipal.

As fórmulas para as taxas de crescimento do IDH e do PIB per capita utilizados neste trabalho foram:

$T C I D H=\left[\left(\frac{I D H}{I D H_{i}}\right)-1\right] * 100$

onde: $\mathrm{TCIDH}=$ taxa de crescimento do IDH municipal no período (\%);

$I D H_{i}=$ IDH do município no ano de 2000;

$I D H=I D H$ do município no ano de 2010.

e $T C P I B C M=\left[\left(\frac{P I B C M}{P I B C M_{i}}\right)-1\right] * 100$

onde: $P I B C M=$ taxa de crescimento do PIB 
per capita municipal no período (\%); PIBCM = PIB per capita do município no ano de 2000;

PIBCM = PIB per capita do município no ano de 2010.

Para analisar se houve tendência à redução ou não das diferenças de IDH e PIB per capita entre os municípios estudados no período avaliado, foi realizada a análise de convergência $(\sigma)$. A convergência $(\sigma)$ caracteriza-se por uma redução da dispersão dos valores dos indicadores ao longo do tempo, o que significaria que o IDHs PIBs per capita das diferentes regiões tende a se aproximar de sua média. Assim, convergência $(\sigma)$ foi testada pela análise do coeficiente de variação $(\mathrm{CV})$, dado pela razão entre o desvio-padrão e a média aritmética dos indicadores.

Quanto ao município de Figueirão, que foi criado em Mato Grosso do Sul no dia $1^{\circ}$ de janeiro de 2005 (PREFEITURA MUNICIPAL DE FIGUEIRÃO, s.d.), os dados estimados de IDH divulgados pelo PNUD para o ano de 2000 foram utilizados; já em relação ao PIB deste município, não foi possível sua comparação entre os anos de 2000 e 2010 em relação ao PIB per capita. Para o Município de Paraíso das Águas, que foi criado em 03 de dezembro de 2009, não havia dados disponibilizados pelas fontes oficiais até o encerramento deste artigo.

\section{O IDH E O PIB PER CAPITA}

\subsection{IDH no Brasil e Mato Grosso do Sul}

Sempre que se estuda um território específico, como um micro, meso, ou macrorregião, busca-se, a partir de suas características, habilidades e vocações para produção de alimentos e adaptação urbana, explicar como os processos relacionados à mobilidade territorial pode afetar o modo de vida de uma comunidade. Dessa maneira, os dados populacionais, especificamente sobre o Índice do Desenvolvimento Humano (IDH), constituem elemento chave para explicação da dinâmica territorial, quando analisamos as estratégias para o desenvolvimento socioespacial das regiões produtivas (SILVA; NASCIMENTO; FREITAS, 2006).

O IDH tem como base o desenvolvi- mento de um grupo de pessoas que vivem em um território específico. Assim, parte do pressuposto de que, para aferir o avanço de uma população, não se deve considerar apenas a dimensão econômica, mas também outras características como: sociais, culturais e políticas que influenciam a qualidade da vida humana (PNUD, s.d.).

Por essa razão, através da análise territorial realizada através do IDH, tem sido possível explicar a integração dos espaços, pelo viés da mobilidade populacional e das economias territoriais. O território, nessa perspectiva, passa a ser concebido e entendido para além das relações de poder quando é, também, considerado uma instância social, ou seja, lócus de exercício de cidadania (SILVA; NASCIMENTO; FREITAS, 2006).

O Índice de Desenvolvimento Humano (IDH) é obtido pelo resultado da síntese dos seguintes indicadores: renda, expectativa de vida, taxa de alfabetização de pessoas com 15 anos ou mais de vida, e taxa de matrícula bruta nos três níveis de ensino, ou seja, a relação entre a população que se encontrava em idade escolar e o número de pessoas matriculadas no ensino fundamental, médio e superior. $\mathrm{O}$ índice varia de zero (nenhum desenvolvimento humano) até um (total de desenvolvimento humano), classificado da seguinte forma: $O$ índice de 0 a 0,499 é considerado muito baixo; 0,5 a 0,599 baixo; 0,6 a 0,699 médio; de 0,7 a 0,799 alto; e de 0,8 a 1,0 muito alto (PNUD, s.d.).

Entre 2000 e 2010, segundo o Programa das Nações Unidas Internacional para o Desenvolvimento (UNDP, s.d.), IDH do Brasil foi de 0,682 para 0,739 .

Quanto aos IDHs estaduais, divulgados pelo Atlas Brasil (PNUD, s.d.), os estados de Alagoas, Maranhão e Piauí, que tinham as piores posições de IDH do país em 2000, foram reclassificados de IDH "Muito Baixo" para IDH "Baixo" em 2010, mas ainda assim se mantiveram nas últimas posições no ranking nacional (figura 1). Já o Distrito Federal, em 2010, alcançou a classificação de IDH "Muito Alto", sendo a única unidade da federação classificada nessa categoria. São Paulo e Santa Catarina foram o $2^{\circ}$ e $3^{\circ}$ colocados, classificados com o IDH "Alto" em 2010. 
IDH 2000

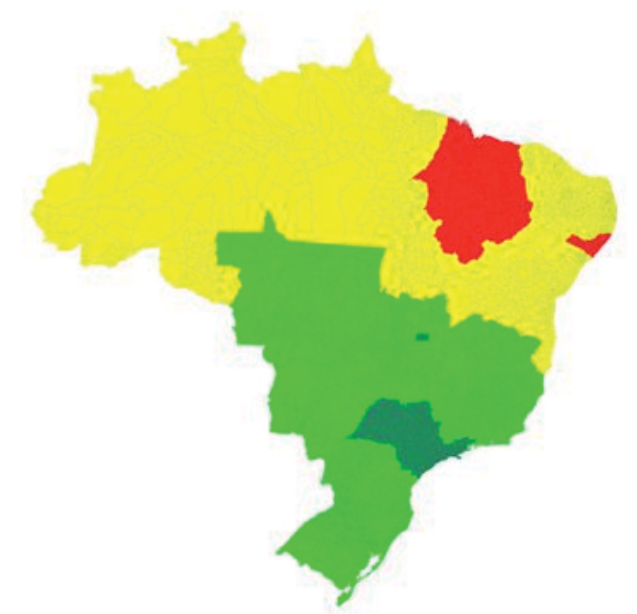

COR LEGENDA

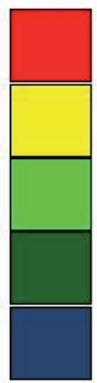

Muito Baixo $[0 ; 0,499]$

Baixo $(0,500 ; 0,599]$

Médio $(0,600 ; 0,699]$

Alto $(0,700 ; 0,799]$

Muito Alto $(0,800 ; 1,000]$

Figura 1 - Classificação do IDH dos estados do Brasil nos anos de 2000 e 2010, segundo PNUD (s.d.).

Fonte: Mapa temático dos autores.

Mato Grosso do Sul ocupou a $10^{\text {a }}$ posição entre as 27 unidades federativas brasileiras em 2010, segundo o IDH do Atlas Brasil. Nesse ranking, o maior IDH foi de 0,824 (Distrito Federal) e o menor foi o de 0,631 (Alagoas).

É importante ressaltar que a média do IDH do Brasil divulgado pelas Nações Unidas Internacional para o Desenvolvimento (UNDP, s.d.), não coincide com o IDH divulgado pelo Atlas Brasil (PNUD, s.d.), pois a esperança de vida ao nascer em nível municipal e estadual do Atlas Brasil são informações coletadas dos censos, através de cálculo indireto utilizando uma adaptação da metodologia de Brass. Já o IDH divulgado pela UNDP usa fontes internacionais que incluem projeções que nem sempre estão com o mes- mo nível de atualização. No caso da renda, a adotada no IDH divulgado pela UNDP, é a renda nacional bruta per capita, que é fruto de um cálculo indireto, obtido no âmbito das contas nacionais, que também geram indicadores como o Produto Interno Bruto e outros indicadores macroeconômicos. Já a adotada no IDH do Atlas Brasil considera a renda das pessoas, calculada a partir dos quesitos sobre rendimentos dos censos demográficos. Portanto são conceitos e fontes diferenciadas. No caso da educação, todas as variáveis que compõem o IDH do Atlas Brasil são diferentes das variáveis que compõem o IDH da UNDP (PNUD, s.d.). O IDH do Atlas Brasil também não coincide com a média aritmética do IDH dos Estados e Municípios, pois considera o tamanho (proporção) dos municípios (figura 2). 


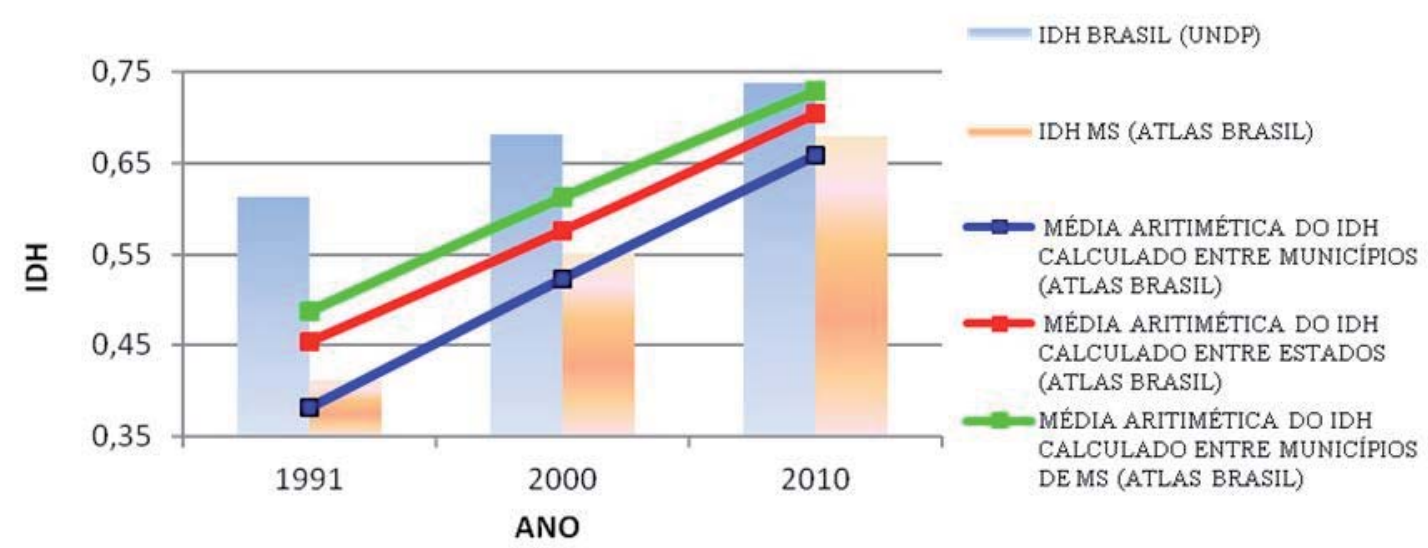

Figura 2 - IDHs oficiais divulgados pelo UNDP (s.d.) e pelo Atlas Brasil PNUD (s.d.) e os IDHs calculados através da média aritmética para o Brasil e para o estado de Mato Grosso do Sul (MS).

Dessa forma, em 2010 o IDH do Brasil divulgado pelo UNDP foi de 0,739 , a média aritmética entre os 27 estados foi de 0,704 , e a média aritmética entre os 5.564 municípios de todo o Brasil foi de 0,659 (figura 2).

O IDH de Mato Grosso do Sul divulgado no Atlas Brasil ficou abaixo do IDH oficial do Brasil, mas, quando verificadas as médias dos IDHs dos Municípios de MS, observou-se que, em 2010, ficaram muito próximas do IDH oficial do Brasil e acima da média do IDHs dos municípios brasileiros (figura 2). Assim é necessária a verificação da metodologia de formação dos IDHs para se analisar, de forma adequada, as diferenças regionais.

\subsection{O IDH dos Municípios de Mato Grosso do Sul}

Em 2010, Mato Grosso do Sul encontrava-se com 79 municípios, com dois municípios fundados entre 2000 e 2010 (Figueirão e Paraíso das Águas), e seu IDH estadual passou de 0,613 em 2000 para 0,729 em 2010 (classificado como IDH alto), com crescimento de 18,92\% (PNUD, s.d.; IBGE, s.d.).

Entre 2000 e 2010, a população de Mato Grosso do Sul cresceu a uma taxa média anual de 1,66\% (2.449.024 pessoas em 2010), ou seja, o crescimento do IDH foi maior que o crescimento populacional. No Brasil, essa taxa de crescimento populacional foi de 1,17\% no mesmo período. Nessa década, a taxa de urbanização do estado passou de $84,08 \%$ para 85,64\% (PNUD, s.d.; IBGE, s.d.).

Em 2000, conforme figura 3, as cidades que tinham os piores IDHs, classificados como "muito baixo" estavam assim dispostas, na região sul do estado: Japorã $(0,399)$, Paranhos $(0,441)$, Tacuru $(0,469)$, Juti $(0,484)$, Novo Horizonte do Sul $(0,494)$ e Itaquirai $(0,498)$; na região central: Dois Irmãos do Buriti $(0,447)$; e na região do baixo pantanal: Caracol $(0,488)$. 


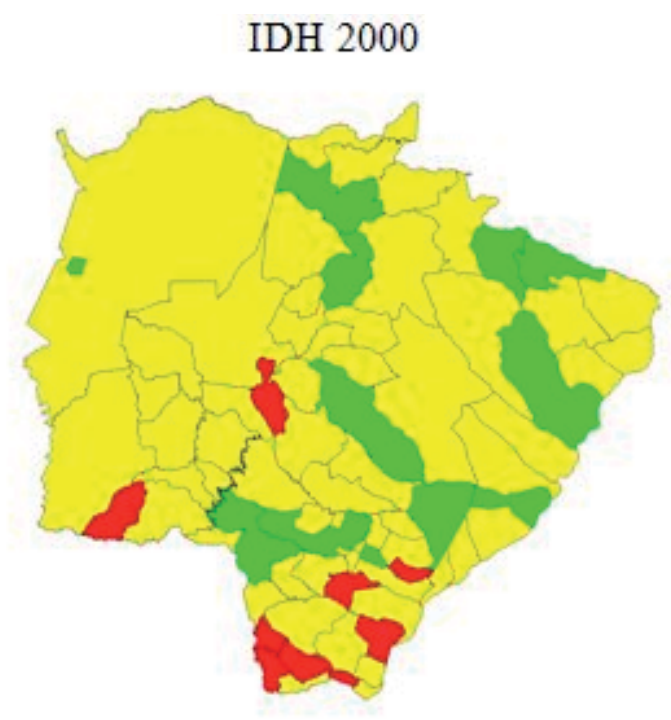

\begin{tabular}{ll} 
COR & LEGENDA \\
\hline & Muito Baixo $[0 ; 0,499]$ \\
\hline & Baixo $(0,500 ; 0,599]$ \\
& Médio $(0,600 ; 0,699]$ \\
& Alto $(0,700 ; 0,799]$ \\
\hline & \\
\hline &
\end{tabular}

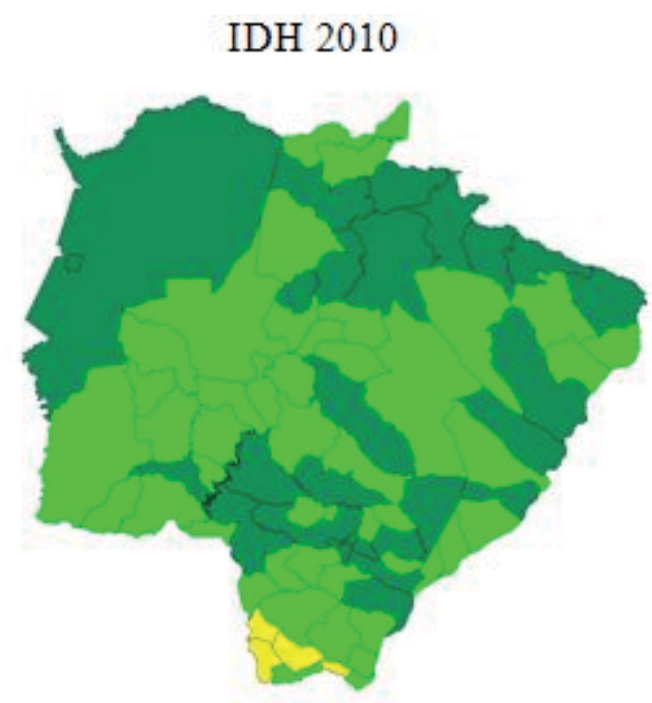

TAXA DE CRESCIMENTO IDH (2000-2010)

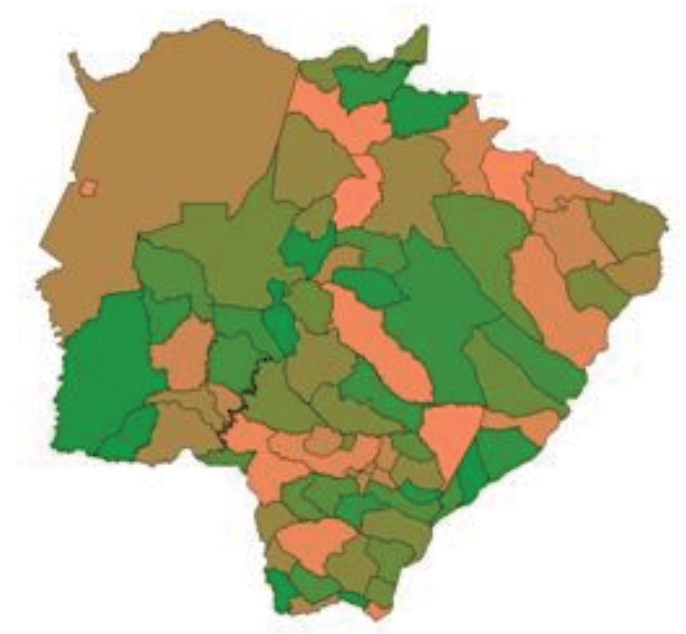

Figura 3 - Classificação e taxa de crescimento do IDH dos municípios no Mato Grosso do Sul nos anos de 2000 e 2010, segundo PNUD (s.d.). Mapa temático dos autores.

Já no ano de 2010, não havia cidades com IDH "muito baixo" no estado, e apenas quatro cidades estavam classificadas com o IDH "baixo", situadas na região sul do estado. São elas: Japorã $(0,526)$, Paranhos $(0,588)$, Coronel Sapucaia $(0,589)$ e Tacuru $(0,593)$, conforme figura 3.

As cidades com os melhores IDHs no ano de 2000 foram (por ordem de classificação): 1ํㅡ Campo Grande (0,673), 2ํSão Gabriel do Oeste $(0,658)$, $3^{\circ}$ Chapadão do Sul $(0,658)$, $4^{\circ}$ Dourados $(0,636)$ e $5^{\circ}$ Três Lagoas $(0,630)$. Essas cidades foram classificadas com IDH "médio" (figura 3).
Em 2010, São Gabriel do Oeste caiu para o $6^{\circ}$ lugar, com Maracaju assumindo a $5^{\mathrm{o}}$ posição no ranking dos IDHs municipais em MS. Dessa forma, segue a classificação do IDH daquele ano: $1^{\circ}$ Campo Grande $(0,784), 2^{\circ}$ Chapadão do Sul $(0,754)$, 3o Dourados $(0,747)$, $4^{\circ}$ Três Lagoas $(0,744)$ e $5^{\circ}$ Maracaju $(0,736)$. Essas cidades foram classificadas com IDH "alto" (figura 3).

Através da figura 3, também é possível visualizar os municípios que tiveram a maior taxa de crescimento do IDH entre 2000 e 2010, classificados da seguinte forma: $1^{\circ}$ 
Dois Irmãos do Buriti (42,95\%), 2oㅡㄹ Batayporã (34,65\%), 3ํ Caracol $(33,68 \%)$, 4 Corguinho $(33,67 \%), 5^{\circ}$ Paranhos (33,33\%), 6o Porto Murtinho (33,93\%), 7o Alcinópolis (32,65\%), $8^{\circ}$ Jaraguari (32,01\%), 9ำ Japorã (31,83\%) e $10^{\circ \circ}$ Novo Horizonte do Sul (31,38\%).

Os 10 municípios que tiveram o menor avanço quanto ao IDH no período de 2000 a 2010 foram: 69ํㅡㅁ Dourados (17,45\%), $70^{\circ}$ Mundo Novo (17,26\%), 71으 Amambaí (16,84\%), $72^{\circ}$ Ponta Porã (16,83\%), 73ํㅡ Campo Grande $(16,49 \%)$, $74^{\circ}$ Ladário $(16,36 \%)$, $75^{\circ}$ Coxim (15,82\%), 76을 Chapadão do Sul $(14,94 \%), 77^{\circ}$ Nova Andradina $(14,44 \%)$ e $78^{\circ}$ São Gabriel do Oeste $(10,79)$.

Nesse sentido, observa-se que os municípios que tinham os menores IDHs no ano 2000 , tiveram os maiores avanços no período pesquisado; enquanto que os municípios com os melhores IDHs em 2000 tiveram um crescimento menos expressivo.

\subsection{PIB per capita no Brasil e em Mato Grosso do Sul}

Entre o início da década de 2000 até a eclosão da crise financeira internacional, em 2008, o Brasil passou por um ciclo de crescimento econômico e inclusão social. Mesmo após 2008, no qual, com exceção de 2010, as taxas de crescimento do produto interno bruto (PIB) foram inferiores às do período anterior, não se observaram retrocessos nos ganhos obtidos em termos de renda per capita e de redução da desigualdade social (CAVALCANTE; DE NEGRI, 2014).

Os fatores que concorreram para esse ciclo de crescimento econômico e inclusão social envolviam as políticas sociais de redistribuição de renda e de valorização do salário mínimo, a expansão do crédito, a incorporação de um grande contingente de população ao mercado de trabalho e de consumo e um cenário externo de crescimento acelerado que impulsionou a valorização de commodities exportadas pelo Brasil (CAVALCANTE; DE NEGRI, 2014).

Porém a desigualdade de renda entre os brasileiros ainda era marcante e, mesmo com a queda consistente desta desigualdade, em 2010 ainda se classificava entre as cinco mais altas do mundo (WORLD BANK, 2015).
A literatura empírica sobre desigualdades de renda tem-se desenvolvido enormemente nas duas últimas décadas. Metodologias exploratórias destacam aspectos como testes de convergência entre localidades pobres com localidades ricas (RESENDE; MAGALHÃES, 2013); Modelos não paramétricos, como matrizes de transição de Markov e superfícies de densidade de Kernel, são utilizadas quando distribuições do PIB per capita não são padronizadas, utilizadas para se observar essas distribuições em corte no tempo (MAGRINI, 2004; ANDERSON; LINTON; LEO, 2012). O índice de Shorrocks é utilizado quando se quer capturar a mobilidade dos níveis regionais de renda (HAMMOND; THOMPSON, 2002), e pela estatística Tau, que captura o grau da estabilidade de rank (REY, 2004). Outros tipos de análises, como a estatística de autocorrelação espacial de Moran I, aliada à econometria espacial para se formar modelos também tem sido muito utilizada, pois enfocam na possibilidade de se verificar se regiões de alta renda estão se consolidando ou se fragmentando ao longo de todo o território nacional em relação ao PIB per capita (REIS et al., 2005).

Em decorrência disso, é de extrema importância analisar o crescimento econômico sob a ótica da "qualidade", e não somente em termos quantitativos; ou seja, deve-se avaliar se esse crescimento tem gerado algum tipo de desenvolvimento social. Assim, é necessário que o crescimento econômico de um país ou de uma região deva vir acompanhado de uma melhora nas condições de vida dos habitantes, especialmente na dos mais pobres.

Uma das formas de verificar se o crescimento veio acompanhado de diminuição da desigualdade social é através do conceito Produto Interno Bruto per capita. Nesse sentido, o PIB per capita compara o crescimento da riqueza produzida em um determinado território, e verifica-se se esta riqueza acompanhou o crescimento populacional nesse local, para que se possa tomar medidas socioeconômicas e diminuir a desigualdade social (CARNEIRO; BAGOLIN, 2000).

A teoria sugere que as instituições e setores econômicos afetam o nível de renda através da distribuição de poder político, da geração de oportunidades econômicas, do estímulo à inovação e à acumulação de capital hu- 
mano, além de outras vias. Assim, diante das grandes disparidades observadas no nível de renda entre as localidades, um grande número de estudos busca explicar quais fatores seriam determinantes para justificar o desempenho econômico. Isto é, quais elementos conduziram algumas localidades a atingir um elevado nível de renda per capita, enquanto outros permaneciam estagnados e/ou em um nível muito inferior. No Brasil, segundo Pereira, Nakabashi e Sachsida (2011), a maior renda per capita municipal é mais do que 190 vezes maior que a menor renda per capita municipal.

Quanto ao aspecto econômico, versões convencionais do modelo neoclássico predizem que, em decorrência do fato de a utilização de fatores de produção estarem sujeitas a rendimentos marginais decrescentes, as rendas per capita das diferentes regiões de um país e, mesmo, dos países tenderiam à equalização, se satisfeita a condição de livre mobilidade de fatores (FERREIRA, 2013).

A base teórica desses modelos de convergência da Teoria Neoclássica, vem do progresso tecnológico, da poupança exógena, taxas de retornos decrescentes dos fatores de produção e tendência a um estado estacionário. Segundo a concepção teórica dos modelos de convergência, se as economias em análise têm preferências e tecnologias parecidas, aquelas mais pobres tendem a apresentar crescimento do seu PIB mais rápido vis-à-vis às mais ricas, reduzindo o gap de desenvolvimento existente entre elas (SPOHR; FREITAS, 2011).

Ocorre, porém, segundo Ferreira (2013), que as evidências empíricas disponíveis em relação às rendas per capita dos países e regiões não confirmam essa previsão, pois a distância que separa os países pobres dos países ricos, no que diz respeito às rendas per capita, não parece estar se reduzindo com o passar do tempo.

No Brasil, por exemplo, apesar dos PIBs per capita estaduais terem melhorado no período estudado (figura 4), observa-se que o gap aumentou entre o Distrito Federal, que possuía o maior PIB per capita, e Maranhão, que tinha menor PIB per capita do país, sendo que a diferença no ano de 2000 entre esses locais eram de $\mathrm{R} \$ 41.344,00$, já no ano de 2010 passou a ser de $\mathrm{R} \$ 51.442,00$. 

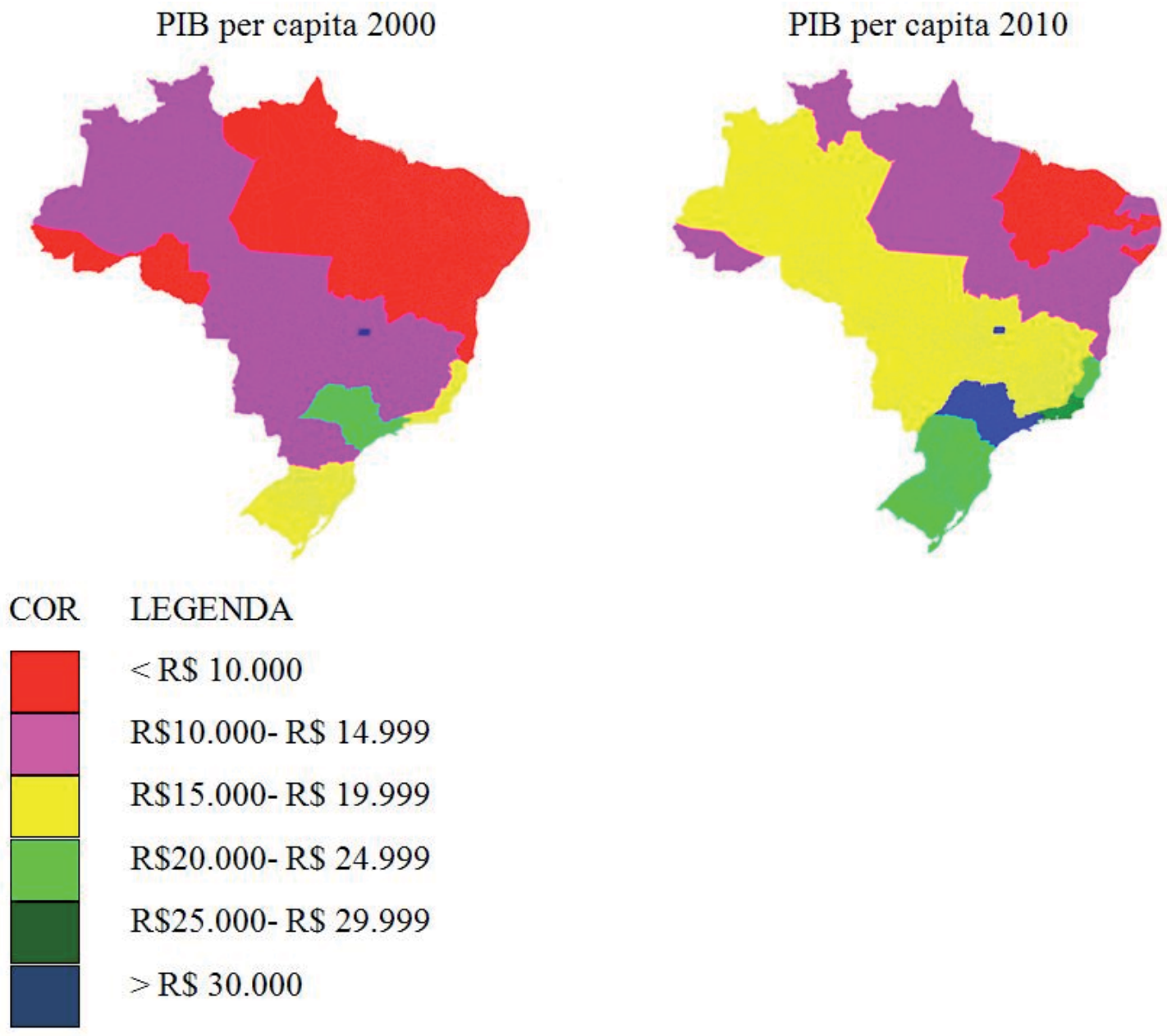

\section{LEGENDA}

$<\mathrm{R} \$ 10.000$

$\mathrm{R} \$ 10.000-\mathrm{R} \$ 14.999$

$\mathrm{R} \$ 15.000-\mathrm{R} \$ 19.999$

$\mathrm{R} \$ 20.000-\mathrm{R} \$ 24.999$

$\mathrm{R} \$ 25.000-\mathrm{R} \$ 29.999$

$>\mathrm{R} \$ 30.000$

Figura 4 - Classificação do Produto Interno Bruto Real per capita dos estados do Brasil nos anos de 2000 e 2010, segundo IBGE (s.d.). Mapa temático dos autores.

A média do PIB per capita entre os estados brasileiros de 2000 a 2010 aumentou de $\mathrm{R} \$ 11.503,00$ para $\mathrm{R} \$ 16.810,00$, sendo os estados com melhores PIBs per capita em 2010 foram: $1^{\underline{0}}$ Distrito Federal $(\mathrm{R} \$ 58.326,00)$, 2o São Paulo (R\$ 30.236,00), 3ํ Rio de Janeiro (R\$ 25.461,00). Já os estados com os piores PIBs per capita em 2010 foram: 25을 Alagoas

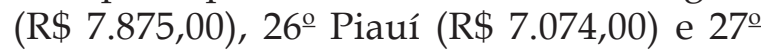
Maranhão (R\$ 6.883,00).

O PIB per capita do estado de Mato Grosso do Sul saltou da $12^{\circ}$ colocação em 2000 (R\$ $10.960,00)$ para a $10^{\circ}$ colocação em 2010 ( $R \$ 17.768,00)$ entre os estados brasileiros, ficando acima da média brasileira no ano de 2010.

O Produto Interno Bruto (PIB) do Mato Grosso do Sul, nos últimos anos, apresentou uma tendência de alta, fato esse que ocorreu também no Brasil. No estado, o PIB real aumentou de R\$22.774.346.519 no ano de 2000 para $\mathrm{R} \$$ 43.514.207.000 em 2010, ou seja, um aumento real de 91,07\% (IBGE, s.d.). Esse comportamento do PIB cria a expectativa de uma melhora nos níveis de bem-estar da população como um todo. Porém o crescimento econômico nem sempre é homogeneamente distribuído, nem dentro, nem entre regiões. Assim, ao se analisar a melhoria dos padrões de vida de determinado local somente sob a ótica do crescimento econômico, podem-se revelar informações que são superficiais e que podem levar a conclusões precipitadas e equivocadas.

\subsection{O PIB per capita dos Municípios de Mato Grosso do Sul}

Em 2000, conforme figura 5, havia 40 cidades com PIB per capita menor R $\$ 10.000,00$, 24 cidades com PIB capita entre $\mathrm{R} \$ 10.000,00$ a R\$ 14.999,00; 9 cidades com PIB per capita 
entre $R \$ 15.000,00$ a $R \$ 19.999,00 ; 1$ cidade com PIB per capita entre R\$20.000,00 a R\$ $24.999,00 ; 2$ cidades com PIB per capita entre R\$25.000,00 a R\$29.999,00; e 1 cidade com PIB per capita acima de $\mathrm{R} \$ 30.000,00$. As cidades com melhores PIBs per capita naquele ano foram: 1ำ Chapadão do Sul (R\$58.326,00),

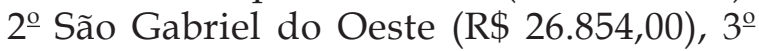
Sonora (R\$25.165,00). Já as cidades com os piores PIBs per capita foram: $75^{\circ}$ Ladário $(\mathrm{R} \$$ $4.386,00), 76^{\circ}$ Paranhos $(\mathrm{R} \$ 4.172,00)$ e $77^{\circ}$ Japorã (R\$ 4.133,00).
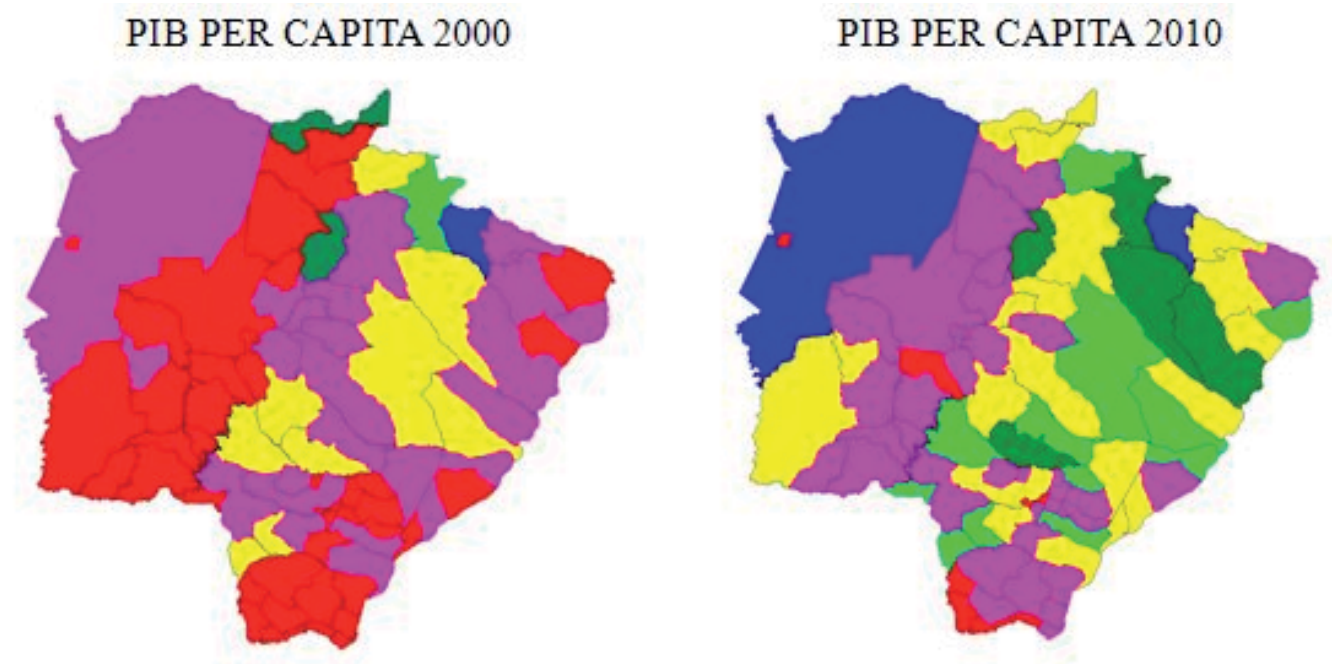

TAXA DE CRESCIMENTO

PIB PER CAPITA (2000-2010)

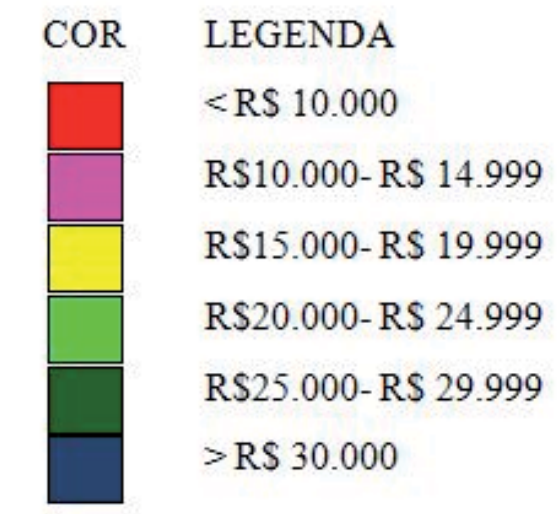

$-31,85 \%$

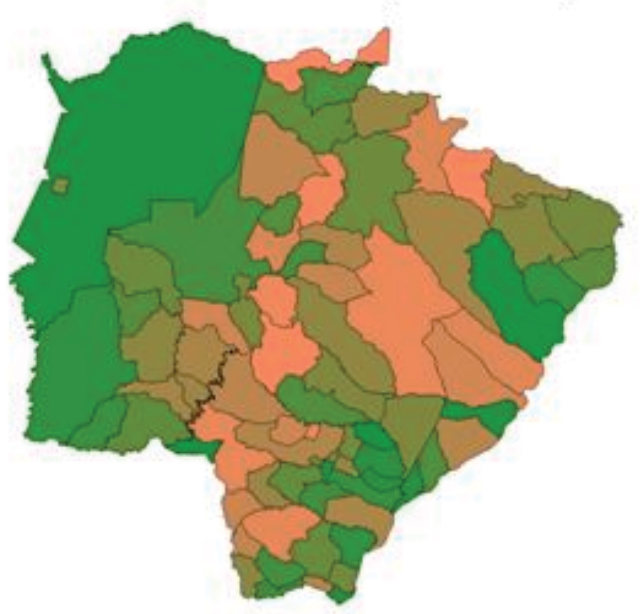

Figura 5 - Classificação e taxa de crescimento do PIB per capita dos municípios de Mato Grosso do Sul nos anos de 2000 e 2010, segundo IBGE (s.d.). Mapa temático dos autores.

Já em 2010, conforme figura 5, havia oito cidades com PIB per capita menor $\mathrm{R} \$ 10.000,00$, 32 cidades com PIB capita entre R $\$ 10.000,00$ a R $\$ 14.999,00 ; 19$ cidades com PIB per capita entre $R \$ 15.000,00$ a $R \$ 19.999,00 ; 12$ cidades com PIB per capita entre R\$20.000,00 a R\$ 24.999,00; cinco cidades com PIB per capita entre $\mathrm{R} \$ 25.000,00$ a $\mathrm{R} \$ 29.999,00$, e duas cida- des com PIB per capita acima de $\mathrm{R} \$ 30.000,00$. As cidades com melhores PIBs per capita naquele ano foram: $1^{\circ}$ Chapadão do Sul (R\$ $34.726,00), 2^{\circ}$ Corumbá (R\$31.327,00), $3^{\circ}$ São Gabriel do Oeste (R\$28.075,00). Já as cidades com os piores PIBs per capita foram: $75^{\circ}$ Coronel Sapucaia (R $\$ 6.807,00), 76^{\circ}$ Paranhos $(\mathrm{R} \$ 6.578,00)$ e $77^{\circ}$ Japorã $(\mathrm{R} \$ 5.926,00)$. 
Através da figura 5 é possível também visualizar os municípios que tiveram a maior taxa de crescimento do PIB per capita entre 2000 e 2010, classificados da seguinte forma: $1^{\circ}$ Antônio João (223,32\%), 2ํㅡㄹ Angélica $(207,49 \%), 3^{\circ}$ Três Lagoas $(167,45 \%), 4^{\circ}$ Corumbá (164,85\%), 5을 Vicentina (163.35\%), 6을 Bataguassu (135,31\%), 7으 Taquarussu $(134.27 \%)$, $8^{\text {ㅇ }}$ Mundo Novo $(125,95 \%)$, 9음 Novo Horizonte do Sul (121,38\%) e 10ำ Japorã $(109,28 \%)$.

Os 10 municípios que tiveram o menor avanço quanto ao PIB per capita no período de 2000 a 2010 foram: $68^{\circ}$ Costa Rica (26,39\%), 69응 Brasilândia (24,96\%), 70ํAmambai (24,89\%), $71^{\circ}$ Ponta Porã (23,68\%), $72^{\circ}$ Ribas do Rio Pardo

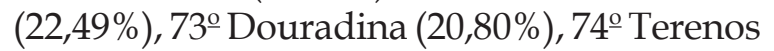
$(19,65 \%)$, 75ํosão Gabriel do Oeste $(4,54 \%)$,
76ºSidrolândia (-3,79\%), 77ํㅡㄹ Chapadão do Sul $(-15,71 \%)$ e $78^{\circ}$ Sonora $(-31,85 \%)$.

$\mathrm{O}$ crescimento negativo desses três últimos municípios citados foi devido ao aumento populacional expressivo no período analisado, tendo superado a média do estado, que foi de $1,17 \%$. Dessa forma, o crescimento populacional foi: Sidrolândia $(79,42 \%)$, Chapadão do Sul (68,54\%) e Sonora (55,43\%).

Em 2000 a média aritmética dos PIBs per capita entre os municípios do MS foi de $R \$ 10.196,00$ (figura 6), sendo que 35 municípios ficaram acima da média, e 42 municípios ficaram abaixo da média. Já em 2010, a média dos PIBs entre os municípios do MS foi de R $\$ 16.348,00$, permanecendo 35 municípios acima da média, e 42 municípios abaixo da média.

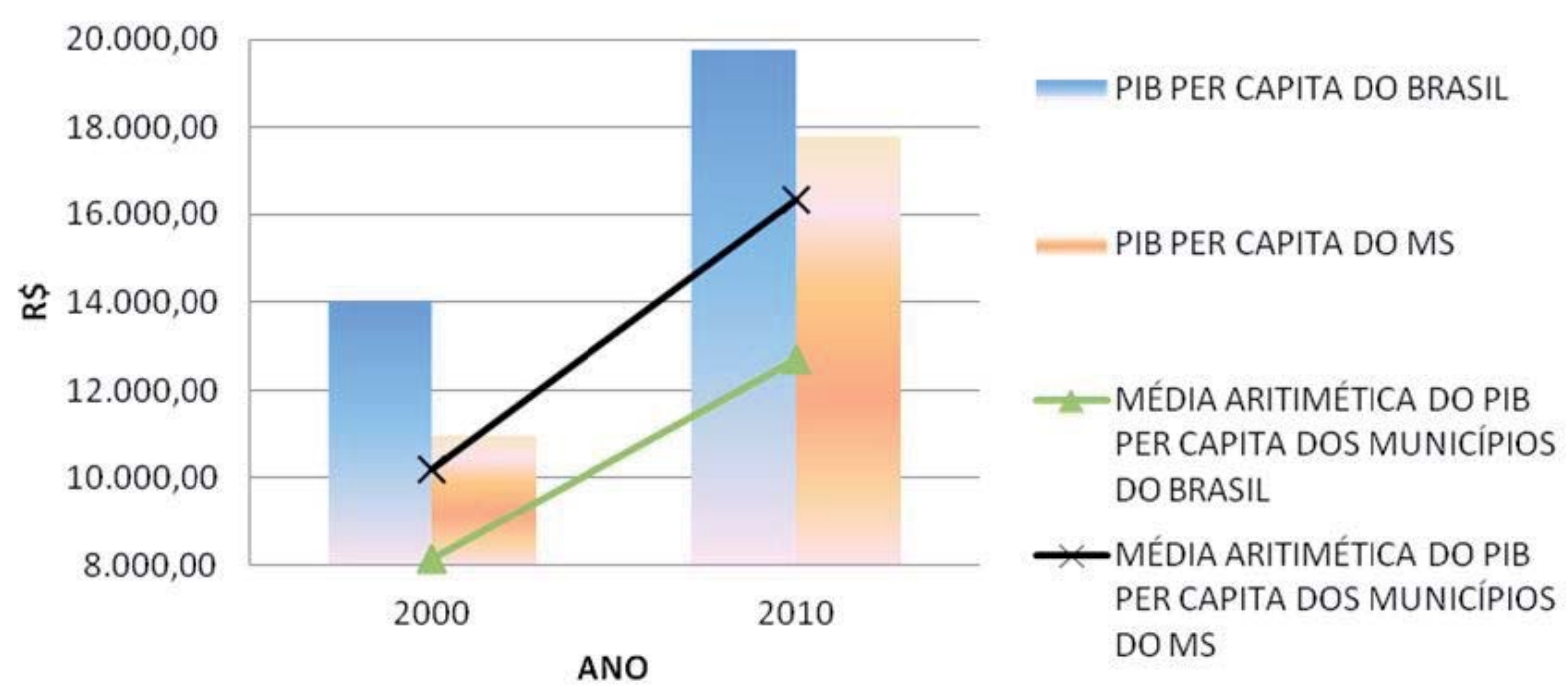

Figura 6 - Produto Interno Bruto per capita real divulgado pelo IBGE (s.d.), do Brasil, do estado de Mato Grosso do Sul, e as médias aritméticas dos municípios do estado e do país.

As médias aritméticas dos PIBs per capita dos municípios foram menores que os PIBs per capita comparados com o do estado e o nacional (figura 6); sendo que, nesse caso, essas diferenças ocorreram porque os PIBs per capita do MS e do Brasil foram calculados considerando as pessoas, suas rendas individualmente, enquanto o PIB per capita municipal divide seu PIB pela soma da renda total das pessoas residentes no município.

\subsection{Análise de convergência dos indicadores}

Quando se trabalha com análise de convergência, é importante ressaltar que, normalmente, a literatura distingue dois tipos de convergência: $\beta$ convergência e $\sigma$ convergência. $\beta$ convergência caracteriza-se por uma relação negativa entre IDH inicial e taxa de crescimento do IDH, o que implica que as economias menos desenvolvidas tendem a desenvolver mais rápido que as 
mais desenvolvidas (VIEIRA; SONAGLIO; CARVALHO, 2009).

Neste trabalho, no entanto, foi realizado apenas o teste $\sigma$ convergência, sendo que a condição suficiente de o convergência é que se verifique uma queda na dispersão dos indicadores municipais em relação à média dos municípios do MS no período avaliado. Assim, valores de zero para $\mathrm{CV}$ significariam uma perfeita igualdade no IDH e PIB per capita entre os municípios.
Nesse sentido, a tabela 01 indica os resultados do teste $\sigma$ convergência. Nota-se que as médias dos IDHs e dos PIBs per capita aumentaram no período estudado, porém os coeficientes de variação diminuíram entre 2000 a 2010; portanto, este resultado indica que houve convergência para IDH e PIB per capita entre os municípios, isto é, que as diferenças nos índices de IDHs e dos PIBs per capita dos municípios de MS tenderam a diminuir no período analisado.

Tabela 1 - Coeficiente de Variação (CV) do IDH e do PIB per capita (R\$) dos municípios de Mato Grosso do Sul no período de 2000 a 2010.

\begin{tabular}{lcccc}
\hline INDICADORES & IDH (2000) & IDH (2010) & PIB PER CAPITA (2000) & PIB PER CAPITA (2010) \\
\hline MÉDIA & 0,551 & 0,680 & $10.598,883$ & $16.348,018$ \\
\hline DESVIO & 0,051 & 0,041 & $5.679,844$ & $5.997,003$ \\
\hline CV $(\%)$ & 9,286 & 6,084 & 53,589 & 36,683 \\
\hline
\end{tabular}

\section{CONSIDERAÇÕES FINAIS}

Observou-se que houve um grande avanço do IDH dos Municípios de MS, sendo que 2010 ficou acima da média do IDHs dos municípios brasileiros, e que não havia cidades com IDH "muito baixo" no estado. Porém, alguns municípios da região sul do estado ainda preocupam, pois apresentaram IDH "baixo", o que requer atenção para políticas direcionadas à educação, saúde e renda.

O PIB per capita do estado de Mato Grosso do Sul e seus municípios aumentaram no período estudado, ficando acima da média brasileira no ano de 2010. Porém houve um crescimento negativo desse indicador para os municípios de Sidrolândia, Chapadão do Sul e Sonora, devido ao aumento populacional que foi superior à média estadual.

A convergência do IDH e do PIB per capita entre 2000 a 2010 foi constatada; ou seja, aqueles municípios que anteriormente tinham os piores indicadores, foram os que mais avançaram no sentido da diminuição das desigualdades sociais e econômicas, mostrando que Mato Grosso do Sul avançou na qualidade de vida de sua população durante essa década e que o mapeamento dos indicadores poderá ajudar a resolver os problemas locais de uma forma mais efetiva.

\section{REFERÊNCIAS}

ANDERSON, G.; LINTON, O.; LEO, T. A polarizationcohesion perspective on cross-country convergence. Journal of economic growth, v. 17, n. 1, p. 49-69, 2012.

CARNEIRO, Douglas Mesquita; BAGOLIN, Izete Pengo. Distribuição da renda do trabalho versus perfil socioeconômico e crescimento nos três Coredes de menor PIB per capita do Rio Grande do Sul nos anos 2000. Indicadores Econômicos FEE, v. 40, n. 1. 2012.

CAVALCANTE, Luiz Ricardo; DE NEGRI, Fernanda. Produtividade no Brasil: uma análise do período recente. Repositório Ipea. 2014. Disponível em: <http://hdl. handle.net/11058/3016>. Acesso em: 3 jul. 2015.

FERREIRA, Afonso Henriques Borges. O debate sobre a convergência de rendas per capita. Nova Economia, v. 5, n. 2, 2013.

FUNDO MONETÁRIO INTENACIONAL (FMI). [s.d.]. Disponível em: <http://www.imf.org/external/pubs/ft/ weo/2015/01/weodata/index.aspx>. Acesso em:7jul. 2015.

HAMMOND, G. W.; THOMPSON, E. Mobility and modality trends in US state personal income. Regional studies, v. 36, p. 275-287, 2002.

INSTITUTO BRASILEIRO DE GEOGRAFIA E ESTATÍSTICA (IBGE). [s.d.]. Disponível em: <www. ibge.gov.br/sidra>. Acesso em: 23 maio 2015.

MAGRINI, S. Regional (di)convergence. Handbook of regional and urban economics, v. 4, p. 2.741-2.796, 2004.

PEREIRA, Ana Elisa Gonçalves; NAKABASHI, Luciano; SACHSIDA, Adolfo. Qualidade das Instituições e PIB per capita nos Municípios Brasileiros. Repositório Ipea. 2011. Disponível em: <http://hdl.handle.net/11058/1496>. Acesso em: 3 jul. 2015. 
PREFEITURA MUNICIPAL DE FIGUEIRÃO. [s.d.]. Disponível em: <http://www.figueirao.ms.gov.br>. Acessado em: 3 jul 2015.

PROGRAMA NACIONAL DAS NAÇÕES UNIDAS PARA O DESENVOLVIMENTO (PNUD). Atlas Brasil. [s.d.]. Disponível em: <www.pnud.org.br>. Acessado em: 23 de maio de 2015.

REIS, E. J.; TAFNER, P.; PIMENTEL, M.; SERRA, R. V.; REIFF, L. O.; MAGALHÃES, K.; MEDINA, M. O PIB dos municípios brasileiros: metodologia e estimativas-1970-1996. Repositório Ipea. 2005. Disponível em: <http:/ / hdl.handle.net/11058/1920>. Acesso em: 3 jul. 2015.

RESENDE, Guilherme Mendes; MAGALHÃES, João Carlos Ramos. Disparidades do produto interno bruto (PIB) per capita no Brasil: uma análise de convergência em diferentes escalas regionais (1970-2008). Repositório Ipea. 2013. Disponível em: <http://hdl.handle. net/11058/1212>. Acesso em: 3 jul. 2015.

REY, S. J. Spatial dependence in the evolution of regional income distributions. Spatial econometrics and spatial statistics. New York: Palgrave Macmillan, 2004.
SILVA, L. A. D. S.; NASCIMENTO, C. D. J.; FREITAS, N. B. Dinâmica Territorial e Índice de Desenvolvimento Humano (IDH) no Semi-árido Baiano: análise das Microrregiões Geográficas de Paulo Afonso e Juazeiro. Feira de Santa, BA: UEFS, 2006.

SPOHR, Gabriela; FREITAS, Clailton Ataídes de. Teste da Convergência do PIB Per capita da Agropecuária no Brasil entre 1980 e 2004. Revista de Economia e Sociologia Rural, v. 49, n. 2, p. 341-368, 2011.

UNITED NATIONS DEVELOPMENT PROGRAMME (UNDP). [s.d.]. Disponível em: <http:/ / hdr.undp.org/ en/countries>. Acesso em: 3 jul 2015.

VIEIRA, Norberto Martins; SONAGLIO, Cláudia Maria; CARVALHO, Fátima Marília Andrade de. Convergência de renda na Amazônia legal: estudo no arco do povoamento adensado. Revista Brasileira de Gestão e Desenvolvimento Regional, v. 4, n. 4, 2009.

WORLD BANK. GINI index. [s.d.]. Disponível em: <http://data.worldbank.org/indicator/si.pov.gini>. Acesso em: 3 jul. 2015. 